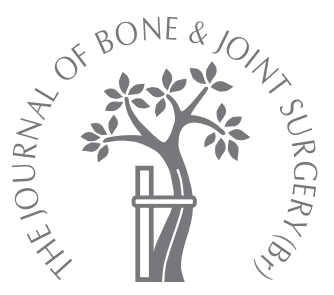

S. I. S. Oussedik, M. B. Dodd, F. S. Haddad

From University College Hospital London, London, United Kingdom

S. I. S. Oussedik, BSc FRCS(Orth), Specialist Registrar

Chelsea and Westminster Hospital, 369 Fulham Road, London SW10 9NH, UK.

M. B. Dodd, BSc, MRCS, MFSEM, Specialist Registrar 58A Niton Street, London SW6 $6 \mathrm{NJ}, \mathrm{UK}$

E. S. Haddad, MCh (Orth), FRCS (Orth), FFSEM,

Consultant Orthopaedic

Surgeon

Department of Trauma and

Orthopaedics

University College Hospital,

235 Euston Road, London NW1

2BU, UK.

Correspondence should be sent to Mr S. I. S. Oussedik; e-mail: sam_ous@hotmail.com

(C)2010 British Editorial Society of Bone and Joint Surgery doi:10.1302/0301-620X.92B9. $23663 \$ 2.00$

$J$ Bone Joint Surg $[\mathrm{Br}]$ 2010;92-B:1222-6.

Received 11 October 2009,

Accepted after revision 22 April 2010

\section{Outcomes of revision total hip replacement for infection after grading according to a standard protocol}

\begin{abstract}
Periprosthetic infection following total hip replacement can be a catastrophic complication for the patient. The treatments available include single-stage exchange, and two-stage exchange. We present a series of 50 consecutive patients with a diagnosis of infected total hip replacement who were assessed according to a standardised protocol. Of these, 11 underwent single-stage revision arthroplasty with no recurrence of infection at a mean of 6.8 years follow-up (5.5 to 8.8). The remaining 39 underwent two-stage revision, with two recurrences of infection successfully treated by a second two-stage procedure. At five years, significant differences were found in the mean Harris Hip Scores (single-stage 87.8; twostage 75.5; $p=0.0003)$ and in a visual analogue score for satisfaction $(8.6 ; 6.9 ; p=0.001)$ between the single- and two-stage groups. Single-stage exchange is successful in eradicating periprosthetic infection and results in excellent functional and satisfaction scores.

Identification of patients suitable for the single-stage procedure allows individualisation of care and provides as many as possible with the correct strategy in successfully tackling their periprosthetic infection
\end{abstract}

Estimates of the incidence of infection following primary hip replacement vary between $1 \%$ and $3 \% .^{1-4}$ The goal of treatment in such cases is to eradicate the infection while ensuring the optimal functional outcome. ${ }^{1,5-7}$

Two-stage revision remains the optimum procedure for eradicating infection, with reported success rates of $80 \%$ to $95 \% .{ }^{1,7-14}$ However, such success comes at a price, with two operations and their associated morbidities, a prolonged hospital stay and reduced morbidity resulting in a limited functional outcome. 1,11,15,16 $^{-1}$ This may be improved with the use of an articulating antibiotic-laden cement spacer ${ }^{1,6,14,15,17-19}$ and a smaller interval between the two stages. ${ }^{6,11,17-19}$ The second stage of a two-stage revision is further complicated by the altered anatomy and loss of planes of dissection and this becomes increasingly difficult with each additional procedure. ${ }^{3,5,10,13,18,20}$ All of these factors also contribute to an increase in cost. ${ }^{1,21,22}$

Single-stage exchange replacement has been proposed as an alternative to a two-stage pro-

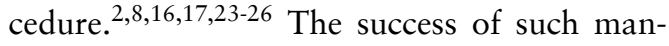
agement has improved significantly over the past two decades with the introduction of antibiotic-loaded cement, better identification of pathogens and their sensitivities, and recognition of the importance of thorough debridement and lavage before reconstruction. ${ }^{1,16,23-26}$ The success rates for eradication range from $35 \%$ to $94 \% .^{8,23-27}$ Associated benefits include a reduction in the length of hospital stay, avoidance of a second procedure with its associated morbidities, improved postoperative mobility and pain, as well as the reduction in cost. ${ }^{1}$ Relative contraindications to a single-stage procedure include significant severe bone loss, an unidentified pathogen and the presence of multiresistant bacteria. 9,23

With the success of single-stage revision now well documented, the identification of cases in which it may be employed becomes more pertinent. Such individualisation of care could lead to an improved outcome by not exposing patients to the extra morbidity and loss of function associated with two-stage procedures while also ensuring eradication of infection. ${ }^{27}$

This study comprised a cohort of patients with an infected hip replacement, treated with strict criteria for single-stage revision. The outcomes were evaluated at a minimum of five years post-operatively.

\section{Patients and Methods}

We prospectively enrolled 50 consecutive patients presenting between 1999 and 2002 with an infected total hip replacement into the 
Table I. Criteria for single- versus two-stage revision

\begin{tabular}{ll}
\hline Single-stage & Two-stage \\
\hline Healthy soft tissues & $\begin{array}{l}\text { Additional compromising } \\
\text { factors }\end{array}$ \\
Minimal bone loss allowing cemented femoral reconstruction & \\
Organism and sensitivities known & \\
\hline
\end{tabular}

Table II. Additional compromising factors considered

\begin{tabular}{ll}
\hline Category & Compromising factors \\
\hline Organism factors & Multiresistant organisms MRSA/MRSE \\
& Polymicrobial infection \\
& Unusual commensals \\
& Unusual resistance profiles \\
& Unidentified infective organisms \\
& Immunosuppression \\
Host factors & Concurrent sepsis \\
& Systemic disease \\
& Re-infection \\
Local factors & Significant bone loss precluding cemented recon- \\
& struction \\
& Significant soft-tissue compromise \\
& Peripheral vascular disease \\
\hline
\end{tabular}

study. All were evaluated by their history; a thorough physical examination, including assessment of local soft tissues; baseline CRP and ESR; hip aspiration and/or biopsy at least four weeks after the end of antibiotic therapy; and plain anteroposterior and lateral radiographs, with additional CT if deemed necessary for further acetabular assessment. The diagnosis of infection was made on the basis of the clinical findings and investigations. All patients were then graded by a standardised protocol based on the criteria previously set out by Haddad et al. ${ }^{18}$ This involves evaluation according to organism(s), and local and systemic factors. The criteria for consideration of single-stage revision are shown in Table I, with additional compromising factors listed in Table II.

Single-stage revision arthroplasty. The infected prosthesis was removed via a posterior approach, using the same incision and a meticulous debridement and lavage carried out, with removal of all cement, membranes, foreign material and potentially infected tissue. All operations were performed by a single surgeon (FSH). Several microbiological samples were taken from the capsule, femur and acetabulum. Antibiotics were withheld until all samples had been taken. Once the surgeon was happy that debridement was complete, all drapes, gowns, gloves and equipment were changed to create a new, sterile environment. The femur was then reconstructed using a cemented femoral component in all cases, with varied fixation of the acetabular component. The cement used was antibiotic-loaded Palacos R (Schering Plough Ltd, Labo nv, Belgium) impregnated with gentamicin. In addition, appropriate antibiotic, determined by known infecting organism sensitivities, was added at a volume of $<5 \%$ of the total weight of cement powder as standard.
Patients received five days of appropriate intravenous antibiotic therapy post-operatively, usually teicoplanin unless otherwise indicated, followed by a minimum of six weeks of oral antibiotics, adjusted if necessary following microbiological review. Antibiotics were prescribed orally after five days whenever possible, and continued until inflammatory markers and nutritional markers (serum albumin and liver function tests) normalised or reached a steady state.

Two-stage revision arthroplasty. The infected prosthesis was removed followed by meticulous debridement and lavage. Multiple tissue samples were taken for microbiological analysis. The drapes and equipment were changed and a temporary articulating, antibiotic-laden cement spacer was implanted. This spacer contained $3 \mathrm{~g}$ of vancomycin and $2 \mathrm{~g}$ of gentamicin per sachet of Palacos $\mathrm{R}$, unless otherwise indicated. The patients received five days of post-operative intravenous antibiotic therapy with teicoplanin. Microbiological culture results were typically available by the fifth day, when antibiotic therapy was altered on the advice of the microbiologists. A total of six weeks' antibiotic therapy, oral if appropriate, was given, with monitoring of the inflammatory markers (CRP and ESR). If these were found to be rising following the first stage, a further aspiration was undertaken with the patient off antibiotics, and further debridement if deemed necessary. The timing of the second stage was dependent on the inflammatory markers reaching a normal or stable level following the end of antibiotic therapy, the rate of wound healing and social factors such as patients who were unwilling or unable to rapidly return for second-stage procedures. The reconstruc- 
Table III. Bacterial culture results

\begin{tabular}{llc}
\hline & \multicolumn{2}{c}{ Number of cases } \\
\cline { 2 - 3 } Cultured organism & Single-stage & Two-stage \\
\hline Staphylococcus aureus & 5 & 3 \\
Corynebacteria & 3 & 2 \\
Enterobacter & 2 & 0 \\
Bacteroides & 1 & 0 \\
Pseudomonas aeruginosa & 0 & 3 \\
Enterococcus & 0 & 2 \\
Coagulase-negative staphylococci & 0 & 19 \\
Methicillin-resistant Staphylococcus & 0 & 6 \\
aureus & & 3 \\
Escherichia coli & 0 & $6{ }^{*}$ \\
Mixed growth & 0 & \\
* four patients with CNS and Gram-negative; one Staphylococcus \\
aureus and enterococcus; one Staph. aureus and fungal growth
\end{tabular}

tion was dictated in each case by the degree of bone loss, with the liberal use of allograft to augment the reconstruction if necessary.

Patients in both groups were followed up for a minimum of five years with serial radiographs, ESR and CRP to assess their progress.

The functional outcome for all patients was evaluated using the Harris Hip score (HHS), ${ }^{28}$ which was recorded preoperatively and at the two- and five-year follow-ups.

Patient satisfaction was assessed by direct questioning, on a visual analogue scale from 1 to 10 .

Statistical analysis was carried out by means of a onetailed two-sample equal variance $t$-test.

\section{Results}

All patients were treated at a single tertiary referral centre. There were 21 men and 29 women with a mean age of 65 (48 to 87 ). The mean follow-up was 6.8 years (5.5 to 8.8), and all patients remain under review. They all had chronic infections and a number had undergone previous revision surgery. For 29 patients this was their first revision, for 18 their second, and for three their third.

After grading, 11 patients were considered appropriate for a single-stage exchange arthroplasty. The remaining 39 underwent two-stage revision. In six cases this was because of mixed infection, with more than one organism cultured; in four cases no organism was identified on initial cultures, although subsequently coagulase-negative staphylococci (CNS) were identified. The results of bacterial culture are summarised in Table III. Evidence of immune compromise was seen in eight patients, with four suffering from rheumatoid arthritis on suppressant therapy and another four requiring steroids.

Seven patients, including three in the immunocompromised group, had a methicillin-resistant Staphylococcus aureus (MRSA) infection and a two-stage procedure was preferred. In 17 patients who would otherwise have been suitable for single-stage exchange, bone loss was too great to allow the use of a cemented femoral component so a two-stage reconstruction was undertaken, using a distal loading uncemented component and bypassing the affected zone.

For the single-stage exchange all femoral components were cemented and six cementless acetabular components were used, reflecting the lead author's (FSH) preference and increasing confidence in single-stage revision over time.

In the two-stage exchange group there were 28 extensively porous-coated femoral components, eight tapered components and three proximal femoral components. On the acetabular side, we used a cage with a cemented polyethylene component in nine cases and a hemispherical porous-coated component in 30 cases, with impaction grafting of morsellised allograft bone in 24 of these.

All of the hips in this series were reconstructed. In the 11 patients who underwent the single-stage procedure there have been no recurrences of infection at a mean of five years' follow-up. In the 39 patients who underwent two-stage revision two had a recurrent infection. Infection was therefore successfully eradicated in $96 \%$ of cases. These two cases were further revised successfully with a second two-stage revision. One further patient required a wound washout ten days after the second stage, having developed a haematoma.

No significant difference was found in the pre-operative HHS $(p=0.24)$. Both groups showed a significant improvement in this score following successful reconstruction, from a mean of 40.5 (16 to 78 ) to a mean of 87.8 (46 to 91 ) in the single-stage group, and 36.5 (12 to 72 ) to 75.5 (40 to 83 ) in the two-stage group. The HHS was found to be significantly higher in the single-stage group at five years $(\mathrm{p}<0.001)$. The improvement at five years was also significantly greater $(p=0.027)$ in the single-stage group. The patient satisfaction scores, showed a significant difference between the two groups at five years, favouring the single-stage procedure. These data are summarised in Table IV. 
Table IV. Harris Hip Scores and visual analogue scale (VAS) satisfaction scores

\begin{tabular}{lccl}
\hline & $\begin{array}{l}\text { Single-stage mean } \\
\text { (range) }\end{array}$ & $\begin{array}{l}\text { Two-stage mean } \\
\text { (range) }\end{array}$ & $\begin{array}{l}\text { p-value } \\
\text { (t-test) }\end{array}$ \\
\hline Pre-operative & $40.5(16$ to 78$)$ & $36.5(12$ to 72$)$ & 0.24 \\
Five years & $87.8(46$ to 91$)$ & $75.5(40$ to 93$)$ & 0.0003 \\
Change & $+47.3(+17$ to +63$)$ & $+39.1(+12$ to +58$)$ & 0.027 \\
Five-year VAS (0 to 10) & $8.6(7$ to 10$)$ & $6.9(4$ to 10$)$ & 0.001 \\
\hline
\end{tabular}

\section{Discussion}

A number of studies have reported on successful outcomes following either single-, ${ }^{2,5,17,29}$ two-stage ${ }^{6,7,11,14,18}$ revision arthroplasty or both ${ }^{9,16}$ for infection. Indeed, one study has reported re-infection rates similar to those seen in revision hip surgery for non-infected joints. ${ }^{7}$

The benefits of a single-stage procedure are clear and are confirmed by our findings. Patients in the single-stage group have an improved functional outcome compared to the two-stage group, although selection bias may undermine the validity of a direct comparison in this study. Additional benefits include the avoidance of a costly second procedure, with a resultant reduction in the duration of hospital stay. ${ }^{8,17}$ These costs may be further reduced by the use of oral antibiotics, ${ }^{30}$ although further analysis is required. $^{21,22}$

Although none of our patients who underwent a singlestage revision suffered re-infection, the numbers in this group were small. Finding larger numbers of patients suitable for the single-stage procedure is difficult, as they are less common within the complex case mix at a tertiary referral centre. Hanssen and Osmon ${ }^{23}$ suggested that the relatively small numbers of patients suitable for a singlestage procedure, with only $11 \%$ of cases in their experience, made it difficult to evaluate its success rate, and therefore concluded that all cases should be treated by twostage revision. In the patients enrolled in our study we identified 11 of 50 , or $22 \%$, who were suitable. Although this is a minority, it is certainly a significant one and, with the benefits of single-stage revision that we have demonstrated, is one that we need to strive to identify effectively and expand. Although direct comparison between single- and two-stage outcomes is difficult, given the non-randomised nature of the study, including those patients treated by the two-stage method gives us an idea of the proportion of cases that might be suitable for single-stage exchange.

The visual analogue score used to measure satisfaction has previously been shown to correlate well with other outcome measures in total joint arthroplasty. ${ }^{31,32}$ Although this indicates a greater satisfaction in the singlestage group, it is essential that patients are not unnecessarily exposed to the possible increased risk of failure of such a strategy. This risk should be minimised by meticulous patient selection. ${ }^{17,26}$ The use of a standardised protocol such as that proposed by McPherson et al ${ }^{27}$ has been shown to aid this process.
During the study we have changed from using a cemented acetabular component in single-stage revision to using uncemented components without any ill effects. However, the choice of femoral implant as dictated by the extent of bone loss remains a limitation to single-stage exchange. The use of antibiotic-loaded cement for femoral fixation has hitherto been considered essential, ${ }^{17,26}$ which limits the choice of prosthesis. In this series, 16 patients underwent two-stage reconstruction because of the presence of bone loss precluding the use of a proximally loading cemented implant. It is to be hoped that the development of new implants which might combine uncemented fixation and infection resistance, either via biofilm disruption or by antibiotic elution, might provide a solution to this problem. ${ }^{33}$

The protocol employed in this series provides a broad and rigorous algorithm that reinforces the importance of a full assessment for each patient and a multidisciplinary approach to their care. The success rate of eradication of infection in 48 of 50 patients $(96 \%)$ is equivalent to the excellent results now reported for two-stage revision. $6,7,9,10,12,13,15,19,34$ It shows that in a selected group of lower risk patients excellent results can be achieved in a single-stage revision, even in units that have traditionally undertaken the two-stage process. All the patients in this study have been followed for a minimum of five years, which would suggest that any reinfection has been picked up.

Although both single- and two-stage revisions are well established in the treatment of infected joint replacement, combining the two techniques in one institution is unusual. This algorithm should help guide the selection of patients who would benefit from a single-stage procedure.

No benefits in any form have been received or will be received from a commercial party related directly or indirectly to the subject of this article.

\section{References}

1. Masterson EL, Masri BA, Duncan CP. Treatment of infection at the site of total hip replacement. Instr Course Lect 1998;47:297-306.

2. Miley GB, Scheller AD Jr, Turner RH. Medical and surgical treatment of the septic hip with one-stage revision arthroplasty. Clin Orthop 1982;170:76-82.

3. Fitzgerald RH Jr, Nolan DR, Ilstrup DM, et al. Deep wound sepsis following total hip arthroplasty. J Bone Joint Surg [Am] 1997;59-A:847-55.

4. Blom AW, Taylor AH, Pattison G, Whitehouse S, Bannister GC. Infection after total hip arthroplasty: the Avon experience. J Bone Joint Surg [Br] 2003;85-B:956-9.

5. Buchholz HW, Elson RA, Engelbrecht $\mathbf{E}$, et al. Management of deep infection of total hip replacement. J Bone Joint Surg [Br] 1981;63-B:342-53.

6. Takahira N, Itoman $\mathbf{M}$, Higashi $\mathbf{K}$, et al. Treatment outcome of two-stage revision total hip arthroplasty for infected hip arthroplasty using antibiotic-impregnated cement spacer. J Orthop Sci 2003;8:26-31. 
7. Haddad FS, Muirhead-Allwood SK, Manktelow AR, Bacarese-Hamilton I. Two-stage uncemented revision hip arthroplasty for infection. J Bone Joint Surg [Br] 2000;82-B:689-94

8. Langlais $\mathbf{F}$. Can we improve the results of revision arthroplasty for infected total hip replacement? J Bone Joint Surg [Br] 2003;85-B:637-40.

9. Tsukayama DT, Estrada R, Gustilo RB. Infection after total hip arthroplasty: a study of the treatment of one hundred and six infections. J Bone Joint Surg [Am] 1996;78-A:512-23

10. Nusem I, Morgan DA. Structural allografts for bone stock reconstruction in twostage revision for infected total hip arthroplasty: good outcome in 16 of 18 patients followed for 5-14 years. Acta Orthop Scand 2006;77:92-7.

11. Colyer RA, Capello WN. Surgical treatment of the infected hip implant: two-stage reimplantation with a one-month interval. Clin Orthop 1994;298:75-9.

12. Wang JW, Chen CE. Reimplantation of infected hip arthroplasties using bone allografts. Clin Orthop 1997;335:202-10.

13. Fehring TK, Calton TF, Griffin WL. Cementless fixation in 2-stage reimplantation for periprosthetic sepsis. J Arthroplasty 1999;14:175-81.

14. Bring GS, Kostamo T, Garbuz DS, Masri BA, Duncan CP. Two-stage revision arthroplasty of the hip for infection using an interim articulated Prostalac hip spacer: a 10- to 15-year follow-up study. J Bone Joint Surg [Br]2009;91-B:1431-7.

15. Hsieh PH, Shih $\mathbf{C H}$, Chang YH, et al. Two-stage revision hip arthroplasty for infection: comparison between the interim use of antibiotic-loaded cement beads and a spacer prosthesis. J Bone Joint Surg [Am] 2004;86-A:1989-97.

16. Salvati EA, Chekofsky KM, Brause BD, Wilson PD Jr. Reimplantation in infection: a 12-year experience. Clin Orthop 1982;170:62-75.

17. Ure KJ, Amstutz HC, Nasser S, Schmalzried TP. Direct-exchange arthroplasty for the treatment of infection after total hip replacement: an average ten-year follow-up. J Bone Joint Surg [Am] 1998;80-A:961-8.

18. Haddad FS, Masri BA, Garbuz DS, Duncan CP. The treatment of the infected hip replacement: the complex case. Clin Orthop 1999;369:144-56.

19. Younger AS, Duncan CP, Masri BA, McGraw RW. The outcome of two-stage arthroplasty using a custom-made interval spacer to treat the infected hip. J Arthroplasty 1997;12:615-23.
20. Crockarell JR, Hanssen AD, Osmon DR, Morrey BF. Treatment of infection with débridement and retention of the components follownig total hip arthroplasty. J Bone Joint Surg [Am] 1998;80-A:1306-13

21. Dreghorn CR, Hamblen DL. Revision arthroplasty: a high price to pay. BMJ 1989;298:648-9.

22. Barrack RL. Economics of revision total hip arthroplasty. Clin Orthop 1995:319:209-14.

23. Hanssen AD, Osmon DR. Assessment of patient selection criteria for treatment of the infected hip arthroplasty. Clin Orthop 2000;381:91-100.

24. Raut VV, Siney PD, Wroblewski BM. One-stage revision of total hip arthroplasty for deep infection: long-term followup. Clin Orthop 1995;321:202-7.

25. Wroblewski BM. One-stage revision of infected cemented total hip arthroplasty. Clin Orthop 1986;211:103-7.

26. Jackson WO, Schmalzried TP. Limited role of direct exchange arthroplasty in the treatment of infected total hip replacements. Clin Orthop 2000;381:101-5.

27. McPherson EJ, Woodson C, Holtom P, et al. Periprosthetic total hip infection: outcomes using a staging system. Clin Orthop 2002;403:8-15.

28. Harris WH. Traumatic arthritis of the hip after dislocation and acetabular fractures: treatment by mold arthroplasty: an end-result study using a new method of result evaluation. J Bone Joint Surg [Am] 1969;51-A:737-55.

29. Raut VV, Siney PD, Wroblewski BM. One-stage revision of infected total hip replacements with discharging sinuses. J Bone Joint Surg [Br] 1994;76-B:721-4.

30. Oussedik SI, Haddad FS. The use of linezolid in the treatment of infected total joint arthroplasty. J Arthroplasty 2008;23:273-8.

31. Bullens PH, van Loon CJ, de Waal Malefijt MC, Laan RF, Veth RP. Patient satisfaction after total knee arthroplasty: a comparison between subjective and objective outcome assessments. J Arthroplasty 2001;16:740-7.

32. de Nies F, Fidler MW. Visual analogue scale for the assessment of total hip arthroplasty. J Arthroplasty 1997;12:416-19.

33. Antoci V Jr, Adams CS, Parvizi J, et al. Covalently attached vancomycin provides a nanoscale antibacterial surface. Clin Orthop 2007;461:81-7.

34. Lieberman JR, Callaway GH, Salvati EA, Pellicci PM, Brause BD. Treatment of the infected total hip arthroplasty with a two-stage reimplantation protocol. 1994;301:205-12 